\title{
Molecular characteristics of the alpha- and beta-tubulin genes of Nosema philosamiae
}

\author{
Feng Zhu ${ }^{1}$, Zhongyuan Shen ${ }^{1,2}, \mathbf{L i}$ Xu ${ }^{1,2}$ and Xijie Guo ${ }^{1,2}$ \\ ${ }^{1}$ Jiangsu University of Science and Technology, Zhenjiang, Jiangsu, China; \\ ${ }^{2}$ Sericultural Research Institute, Chinese Academy of Agricultural Sciences, Zhenjiang, Jiangsu, China
}

\begin{abstract}
Microsporidia are intracellular parasites of insects and other higher eukaryotes. The microsporidian Nosema philosamiae Talukdar, 1961 was isolated from the eri silkworm, Philosamia cynthia ricini Grote. In the present study, alpha- and beta-tubulin genes from $N$. philosamiae were characterized. The identity analysis of nucleotide and amino acid sequences indicated high similarity with species of Nosema Nägeli, 1857 sensu lato (nucleotide sequences, $\geq 96.0 \%$; amino acid sequences, $\geq 99.0 \%$ ). However, the tubulin genes of $N$. philosamiae share low sequence similarity with that of $N$. ceranae Fries, Feng, da Silva, Slemenda et Pieniazek, 1996 (strain BRL01) and a Nosema/Vairimorpha species. Phylogenies based on alpha-, beta- and combined alpha- plus beta-tubulin gene sequences showed that N. philosamiae, along with the true Nosema species, forms a separate clade with a high bootstrap value, with $N$. ceranae BRL01 forming a clade of its own. The results indicated that the alpha- and beta-tubulin sequences may be useful as a diagnostic tool to discriminate the true Nosema group from the Nosema/Vairimorpha group.
\end{abstract}

Keywords: microsporidia, Nosema group, pairwise distance, phylogeny, sequence similarity

This article contains supporting information (Table S1-S5) online at http://folia.paru.cas.cz/suppl/2013-60-5-411.pdf

Microsporidia is a large group of organisms characterized by a highly reduced and compact genome. Molecular karyotype analysis and genome sequencing of several microsporidian species indicate that the human parasite Encephalitozoon intestinalis Cali, Kotler et Orenstein, 1993 has the smallest known genome among eukaryotes (2.3 Mbp) and that many other species, such as Nosema locustae Canning, 1953, Enterocytozoon bieneusi Desportes, 1985, Spraguea lophii Doflein, 1898, have genomes smaller than $10 \mathrm{Mbp}$ (Biderre et al. 1994, Peyretaillade et al. 1998, 2011, Katinka et al. 2001). Although analyses involving SSU rRNA (Vossbrinck et al. 1987) and the elongation factors EF-1 $\alpha$ and EF-2 (Kamaishi et al. 1996a, 1996b) place these organisms in one of the most basal eukaryotic lineages, recent molecular phylogenetic studies support the placement of these eukaryotes amongst fungi, with a probable zygomycete ancestor (Lee et al. 2008a, Texier et al. 2010)

The tubulin gene family consists of six distinct, but highly conserved subfamilies that possess alpha-, beta-, gamma-, delta-, epsilon-, and zeta-tubulins, each defined by sequence conservation and a wide distribution among eukaryotes (McKean et al. 2001). Heterodimers of alphaand beta-tubulin proteins are the major components of microtubules, which in turn are central to the composition of eukaryotic cilia, flagella, mitotic spindles and the cy- toskeleton. Microsporidians have only alpha-, beta-, and gamma-tubulin genes. Eukaryotic alpha- and beta-tubulin proteins have been extensively studied, because they are the most abundant proteins in eukaryotic cells and their evolution may have paralleled that of the nucleus (McKean et al. 2001). The beta-tubulin gene is fairly conserved, with higher amino acid similarity $(>60 \%)$ between the most distantly related lineages (Juuti et al. 2005).

Relationships among the taxa of microsporidians have been inferred primarily from analyses of small subunit rRNA (SSU rRNA) sequences (Vossbrinck and DebrunnerVossbrinck 2005). However, data on recent phylogenies based on alpha- and beta-tubulin protein-coding genes have enabled to carry out more robust phylogenetic analyses (Keeling et al. 2000, Keeling 2003, Akiyoshi et al. 2007, Lee et al. 2008b, Johny et al. 2009, Haag et al. 2011).

The microsporidium Nosema philosamiae Talukdar, 1961 was isolated from the eri silkworm, Philosamia cynthia ricini Grote, in Zhenjiang City, Jiangsu Province, China. This species has been the key factor in obstructing the development of sericulture in China. Studies on the complete rRNA genes and morphological characteristics indicate that this species is closely related to the species of 'true' Nosema (see Zhu et al. 2010).

In the present study, alpha- and beta-tubulin genes of $N$. philosamiae were cloned partially; the nucleotide se- 
quences were compared with corresponding sequences of other microsporidian species for which such data were available, especially species of Nosema Nägeli, 1857.

\section{MATERIALS AND METHODS}

\section{Materials and reagents}

The spores of Nosema philosamiae were isolated from eri silkworms and preserved at the Sericultural Research Institute, Chinese Academy of Agricultural Sciences (SRI-CAAS). LATaq enzyme, restriction enzymes, pMD18-T vectors and T4 DNA ligase were purchased from Takara Company (Takara Bio Inc., Shiga-ken, Japan).

\section{DNA isolation, PCR amplification and sequencing}

Genomic DNA was extracted from the microsporidian as described by Dong et al. (2010). The sequences of the primers (5'-TCCGAATTCARGTNGGNAAYGCNGGYTGGGA-3' - forward and 5'-CGCGCCATNCCYTCNCCNACRTACCA-3' - reverse) used for the amplification of the alpha-tubulin gene were from the study by Lee et al. (2008). The primers (5'-GTAGGAGGAAAGTTCTGGGAG-3' - forward and 5'-TCCTTCACCAGTGTACCAGTG-3' - reverse) used for the amplification of beta-tubulin gene were designed using Premier Primer 5.0 software based on the conserved 5' and $3^{\prime}$ end regions of beta-tubulin genes of four Nosema species, i.e. N. bombycis Nägeli, 1857; N. plutellae Ku, Wang, Tsai, Tzeng et Wang, 2007; Nosema sp. of Tsai, Lo, Soichi et Wang, 2003 (strain PX1); and N. spodopterae Tsai, Lo, Soichi et Wang, 2003.

The PCR protocol followed was as follows: initial denaturation at $95^{\circ} \mathrm{C}$ for $5 \mathrm{~min}$, followed by 30 cycles, each comprising heating for $30 \mathrm{sec}$ at $95^{\circ} \mathrm{C}, 30 \mathrm{sec}$ at $50^{\circ} \mathrm{C}$, and $1 \mathrm{~min} 30 \mathrm{sec}$ at $72{ }^{\circ} \mathrm{C}$, and final extension at $72^{\circ} \mathrm{C}$ for $10 \mathrm{~min}$. The amplified products were subjected to $1.0 \%$ agarose gel electrophoresis, cloned into pMD18-T vectors (Takara Bio Inc., Shiga-ken, Japan), and then sequenced.

\section{Data analysis}

The length and GC content of partial alpha- and beta-tubulin gene sequences of $N$. philosamiae were analysed using the Editseq program of the DNAStar software package (DNASTAR, Madison, Wisconsin, USA). The identity and pairwise distance of the common sequences of selected microsporidian species were analysed using the MegAlign program (DNASTAR, Madison, Wisconsin, USA). The alpha- and beta-tubulin gene sequences were deduced in terms of amino acid sequences by using the Genetyx software (Software Development, Tokyo, Japan).

\section{Phylogenetic analysis}

The nucleotide sequences obtained in the present study and those for microsporidian species compared, including those for 14 alpha-tubulin genes and 15 beta-tubulin genes, were aligned with the Clustal X 1.83 software (Thompson et al. 1997). Phylogenetic trees were constructed with nucleotide sequences of protein-coding genes using the MEGA 5 software package (Tamura et al. 2011). The evolutionary history was inferred by using the maximum likelihood (ML) and maximum parsimony (MP) methods. The bootstrap consensus tree inferred from 1000 replicates was used to represent the evolutionary history of the taxa analysed. Branches corresponding to partitions reproduced in less than $50 \%$ bootstrap replicates were collapsed. The percentage of replicate trees in which the associated taxa clustered together in the bootstrap test (1000 replicates) is shown next to the branches. First and second codon positions were included, whereas the third position was excluded. Trachipleistophora hominis Hollister, Canning, Weidner, Field, Kench et Marriott, 1996 was used as the outgroup.

\section{RESULTS}

\section{Characterization of tubulin genes}

New alpha- and beta-tubulin partial gene sequences from Nosema philosamiae have been deposited in GenBank (accession numbers are shown in Table S1). The N. philosamiae alpha-tubulin partial gene contained an open reading frame of $1198 \mathrm{bp}$ with a GC content of $36.5 \%$ and shared high sequence similarity with true Nose$m a$ species (nucleotide sequences, $N$. bombycis $-96.5 \%$, N. spodopterae $-96.5 \%$, N. plutellae - 97.0\%, and Nosema sp. PX1 - 96.4\%; amino acid sequences, $N$. bombycis $-100 \%$, N. spodopterae $-100 \%, N$. plutellae $-100 \%$ and Nosema sp. PX1 - 99.5\%) (Tables S2, S3).

However, the alpha-tubulin gene of $N$. philosamiae shares low sequence similarity with that of the $N$. ceranae BRL01 Fries, Feng, da Silva, Slemenda et Pieniazek, 1996, a Nosema/Vairimorpha species: $77.8 \%$ identity of the nucleotide sequences and $89.2 \%$ identity of the amino acid sequences.

The partial gene of $N$. philosamiae beta-tubulin contained an open reading frame of $1161 \mathrm{bp}$, with a GC content of $36.1 \%$. The percent identity of pairwise comparisons based on beta-tubulin nucleotide sequences and amino acid sequences also revealed a high similarity (nucleotide sequences $\geq 96.0 \%$; amino acid sequences $\geq 99.0 \%$ ) among $N$. philosamiae, $N$. bombycis, $N$. spodopterae, Nosema sp. PX1 and N. plutellae (Tables S4, S5). However, the nucleotide sequences showed $80.3 \%$ identity with $N$. ceranae BRL01 and the amino acid sequences showed $91.1 \%$ identity with $N$. ceranae BRL01.

\section{Phylogenetic trees constructed from tubulin gene sequences}

The ML and MP phylogenies inferred from the partial alpha-tubulin sequence alignment are based on the nucleotide alignment of 14 microsporidian sequences (Fig. 1). $N$. philosamiae, along with $N$. bombycis, $N$. spodopterae, $N$. plutellae and Nosema sp. PX1, forms a separate clade with a strong bootstrap value ( $>99 \%)$, with $N$. ceranae BRL01 forming a clade of its own. This was further supported by genetic distances, i.e. the pairwise distance of alpha-tubulin nucleotide sequences between $N$. philosamiae, N. bombycis, N. spodopterae, N. plutellae and Nose$m a$ sp. PX1 varied from 0.013 to 0.037 and the pairwise distance of alpha-tubulin nucleotide sequences between $N$. philosamiae and $N$. ceranae BRL01 was found to be 0.264 (Table S2). The pairwise distance of alpha-tubulin amino acid sequences between $N$. philosamiae, N. bombycis, N. spodopterae, N. plutellae, and Nosema sp. PX1 varied from 0 to 0.005 and the pairwise distance of alpha- 


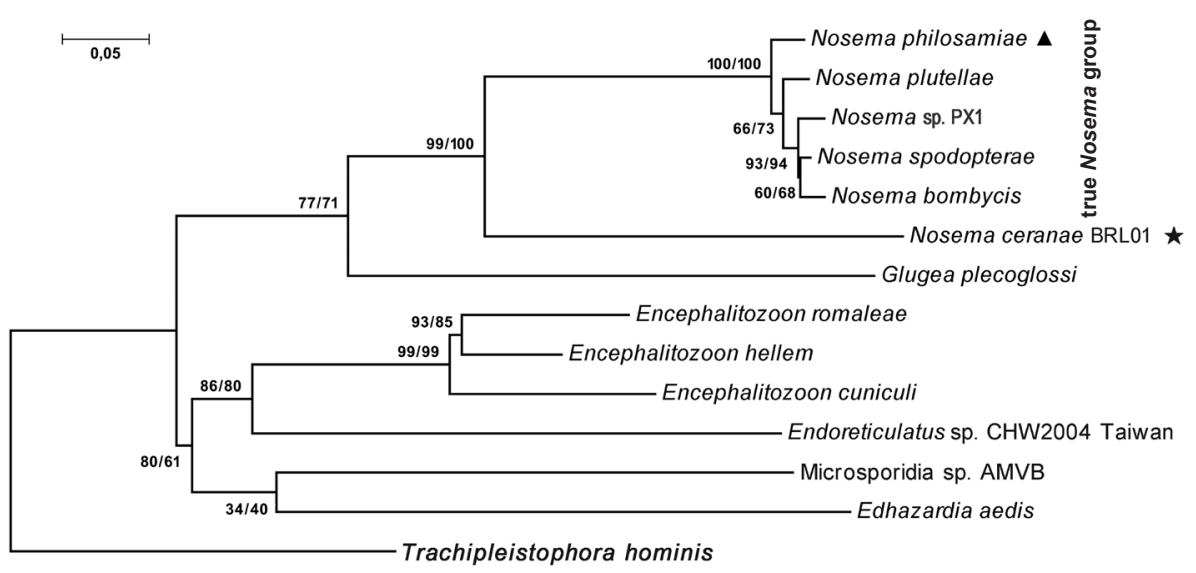

Fig. 1. The maximum likelihood phylogenetic tree based on alpha-tubulin gene sequences. The bootstrap values of MP/ML for 1000 replicates are indicated at nodes. Trachipleistophora hominis was used as outgroup. Nosema philosamiae is indicated by triangle ( $\mathbf{\Delta}$ ) and $N$. ceranae BRL01 by pentagrams $(\star)$.

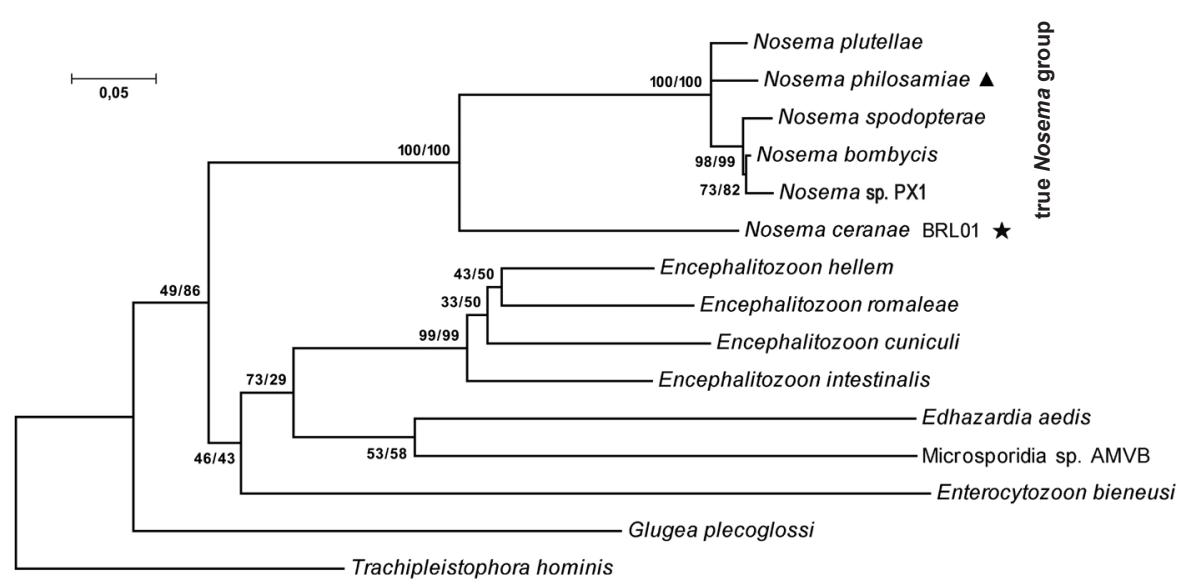

Fig. 2. The maximum likelihood phylogenetic tree based on betha-tubulin gene sequences. The bootstrap values of MP/ML for 1000 replicates are indicated at nodes. Trachipleistophora hominis was used as outgroup. Nosema philosamiae is indicated by triangle $(\boldsymbol{\Delta})$ and $N$. ceranae BRL01 by pentagrams ( $\star$ ).

tubulin amino acid sequences between $N$. philosamiae and N. ceranae BRL01 was found to be 0.116 (Table S3).

The ML and MP phylogenies inferred from the partial beta-tubulin sequence alignment, based on 15 taxa, are shown in Figure 2. The beta-tubulin phylogeny shares many branches in common with that of alpha-tubulin. $N$. philosamiae and four species of the 'true' Nosema clustered reproducibly with a high bootstrap support of $100 \%$. Nosema ceranae BRL01 formed a clade of its own with high bootstrap support (100\%). The ML and MP phylogenies inferred from the combined alpha- and beta-tubulin sequence alignment include 13 taxa (Fig. 3). The combined analysis also indicated a similar relationship between the 'true' Nosema species and N. ceranae BRL01.

\section{DISCUSSION}

The differential diagnosis of Nosema species was based on subtle differences in overlapping characteristics such as spore size, number of nuclei per cell, type of cell division, microsporidium-host relationships, and primary site of infection. However, closely related species are often difficult to differentiate using morphological criteria alone (Wittner and Weiss 1999).

Recently, phylogenetic analysis based on the rRNA sequences suggested that the species that should be placed in Nosema (= 'true' Nosema species) should be separated from the Nosema/Vairimorpha group (Baker et al. 1994, Tsai et al. 2003, 2005, Kyei-Poku et al. 2008). The organization of the rRNA gene of 'true' Nosema species is 5'-LSU-ITS-SSU-3', such as that in N. bombycis, N. spodopterae, $N$. plutellae, $N$. antheraeae and $N$. philosamiae - Huang et al. (2004) Tsai et al. (2005), Wang et al. (2006), Ku et al. (2007), Zhu et al. (2010).

In contrast, Nosema species in the Nosema/Vairimorpha group, which includes N. apis Zander, 1909, N. ceranae and $N$. thomsoni Wilson et Burke, 1971, have the typical rRNA gene subunit arrangement (5'-SSU-ITS-LSU-3') (Gatehouse and Malone 1998, Huang et al. 2008, Kyei-Poku et al. 2008). 


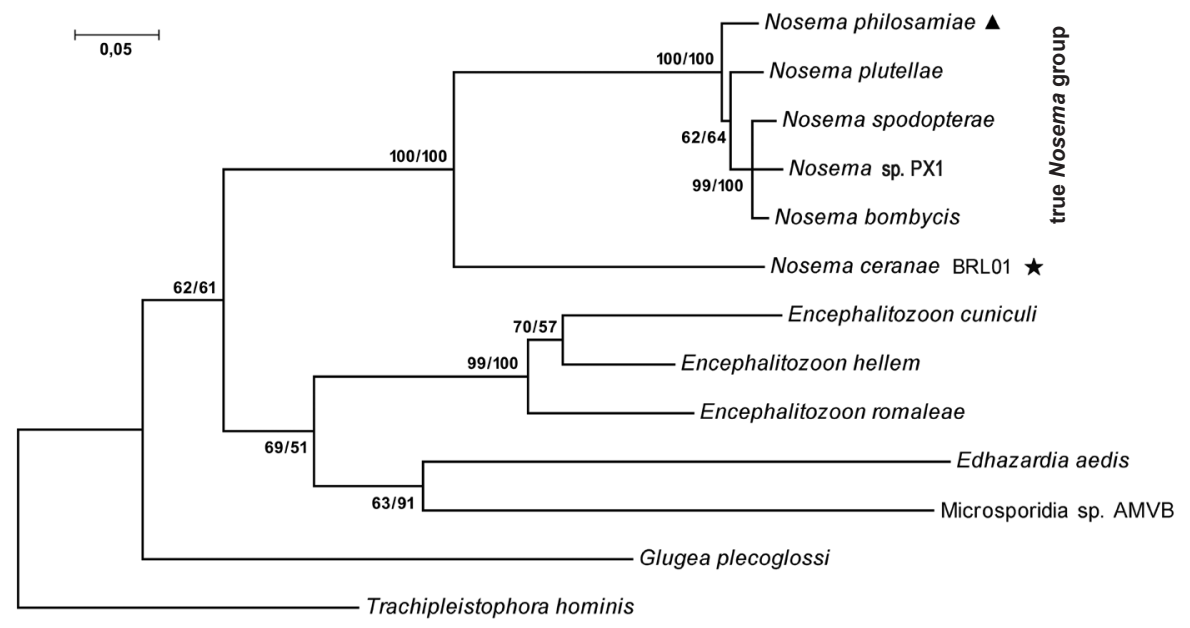

Fig. 3. The maximum likelihood phylogenetic tree based on combined alpha- and betha-tubulin gene sequences. The bootstrap values of MP/ML for 1000 replicates are indicated at nodes. Trachipleistophora hominis was used as outgroup. Nosema philosamiae is indicated by triangle $(\boldsymbol{\Delta})$ and $N$. ceranae BRL01 by pentagrams $(\star)$.

In addition, the 'true' Nosema species, which infect only butterflies (Lepidoptera) are more closely related to each other than they are to other Nosema species (Nosema species in the Nosema/Vairimorpha group) that infect non-lepidopteran hosts (Baker et al. 1994, Zhu et al. 2011). Nageswara Rao et al. (2004) indicated that the distinct grouping of Nosema species that infect only lepidopteran hosts might be caused by the coevolution of the lepidopteran-infecting Nosema species with their host group over time.

In the present study, phylogenies based on alpha-, betaand combined alpha- plus beta-tubulin nucleotide data were in agreement with the relationships that have been proposed on the basis of analyses of rRNA sequences (Zhu et al. 2010). The sequence identity of alpha- and beta-tubulin genes indicated that $N$. philosamiae shares high sequence similarity with the other true Nosema species (nucleotide sequences $\geq 96.0 \%$; amino acid sequences $\geq 99.0 \%$ ), except for $N$. ceranae BRL01.

We found that the tubulin gene/protein sequences of $N$. ceranae BRL01 share low similarity (alpha-tubulin gene $-77.4-78.0 \%$ identity of the nucleotide sequences and $89.2 \%$ identity of the amino acid sequences; beta-tubulin gene $-79.9-81.1 \%$ identity of the nucleotide sequences and $90.6-91.1 \%$ identity of the amino acid sequences) with those of five other Nosema species, which share high se- quence similarity (alpha-tubulin gene, 96.4-98.7\% identity of the nucleotide sequences and $99.5-100 \%$ identity of the amino acid sequences, beta-tubulin gene, $96.0-98.7 \%$ identity of the nucleotide sequences and $98.2-100 \%$ identity of the amino acid sequences) with each other (Tables S2-S5).

The sequence analysis of alpha- and beta-tubulin gene appears to provide substantive evidence for the argument that Nosema species should be properly separated into two groups ('true' Nosema-species group and Nosema/Vairimorpha group) (Baker et al. 1994, Tsai et al. 2003, 2005, Dong et al. 2010, Kyei-Poku et al. 2011, Zhu et al. 2011).

In conclusion, the sequence identity and phylogenetic analysis based on alpha-, beta- and combined alpha- plus beta-tubulin gene sequences have indicated that $N$. philosamiae is a member of the 'true' Nosema group. Moreover, the alpha- and beta-tubulin sequences may be useful as a diagnostic tool to discriminate the 'true' Nosema group from the Nosema/Vairimorpha group.

Acknowledgments. This work was supported by the National Basic Research Program of China (Grant No. 2012CB114600) and the earmarked fund for Modern Agro-industry Technology Research System. We are grateful to everyone who generously provided us with access to the software used in this work. We also thank all our partners and laboratory members for their kind help and criticism. Finally, we are particularly grateful to the reviewers' comments to the manuscript.

\section{REFERENCES}

Akiyoshi D.E., Weiss L.M., Feng X., Williams B.A., Keeling P.J., Zhang Q., Tzipori S. 2007: Analysis of the beta-tubulin genes from Enterocytozoon bieneusi isolates from a human and rhesus macaque. J. Euk. Microbiol. 54: 38-41.

Baker M.D., Vossbrinck C.R., Maddox J.V., Undeen A.H. 1994: Phylogenetic relationships among Vairimorpha and Nosema species (Microspora) based on ribosomal RNA sequence data. J. Invertebr. Pathol. 64: 100-106.
Biderre C., Pages M., Metenier G., David D., Bata J., PrenSier G., Vivares C.P. 1994: On small genomes in eukaryotic organisms: molecular karyotypes of two microsporidian species (Protozoa) parasites of vertebrates. C. R. Acad. Sci. III. 317: 399-404.

Dong S., Shen Z., Xu L., Zhu F. 2010: Sequence and phylogenetic analysis of SSU rRNA gene of five microsporidia. Curr. Microbiol. 60: 30-37. 
Gatehouse H.S., Malone L.A. 1998: The ribosomal RNA gene region of Nosema apis (Microspora): DNA sequence for small and large subunit rRNA genes and evidence of a large tandem repeat unit size. J. Invertebr. Pathol. 71: 97-105.

Haag K.L., Larsson J.I., Refardt D., Ebert D. 2011: Cytological and molecular description of Hamiltosporidium tvaerminnensis gen. et sp. nov., a microsporidian parasite of Daphnia magna, and establishment of Hamiltosporidium magnivora comb. nov. Parasitology 138: 447-462.

Huang W.F., Bocquet M., Lee K.C., Sung I.H., Jiang J.H., CHEN Y.W., WANG C.H. 2008: The comparison of rDNA spacer regions of Nosema ceranae isolates from different hosts and locations. J. Invertebr. Pathol. 97: 9-13.

Huang W.F., Tsai S.J., Lo C.F., Soichi Y., Wang C.H. 2004: The novel organization and complete sequence of the ribosomal RNA gene of Nosema bombycis. Fungal. Gen. Biol. 41: 473-481.

Johny S., Larson T.M., Solter L.F., Edwards K.A., Whitman D.W. 2009: Phylogenetic characterization of Encephalitozoon romaleae (Microsporidia) from a grasshopper host: relationship to Encephalitozoon spp. infecting humans. Infect. Gen. Evol. 9: 189-195.

Juuti J.T., Jokela S., Tarkka M.T., Paulin L., Lahdensalo J. 2005: Two phylogenetically highly distinct beta-tubulin genes of the basidiomycete Suillus bovinus. Curr. Gen. 47: 253-263.

Kamaishi T., Hashimoto T., Nakamura Y., Masuda Y., Nakamura F., Окаmoto K., Shimizu M., Hasegawa M. 1996a: Complete nucleotide sequences of the genes encoding translation elongation factors 1 alpha and 2 from a microsporidian parasite, Glugea plecoglossi: implications for the deepest branching of eukaryotes. J. Biochem. 120: 1095-1103.

Kamaishi T., Hashimoto T., Nakamura Y., Nakamura F., Murata S., Okada N., Okamoto K., Shimizu M., Hasegawa M. 1996b: Protein phylogeny of translation elongation factor EF-1 alpha suggests microsporidians are extremely ancient eukaryotes. J. Mol. Evol. 42: 257-263.

Katinka M.D., Duprat S., Cornillot E., Metenier G., Thomarat F., Prensier G., Barbe V., Peyretaillade E., Brottier P., Wincker P., Delbac F., El Alaoui H., Peyret P., Saurin W., Gouy M., Weissenbach J., Vivares C.P. 2001: Genome sequence and gene compaction of the eukaryote parasite Encephalitozoon cuniculi. Nature 414: 450-453.

Keeling P.J. 2003: Congruent evidence from alpha-tubulin and beta-tubulin gene phylogenies for a zygomycete origin of microsporidia. Fung. Gen. Biol. 38: 298-309.

Keeling P.J., Luker M.A., Palmer J.D. 2000: Evidence from beta-tubulin phylogeny that microsporidia evolved from within the fungi. Mol. Biol. Evol. 17: 23-31.

Ku C.T., Wang C.Y., Tsai Y.C., Tzeng C.C., Wang C.H. 2007: Phylogenetic analysis of two putative Nosema isolates from cruciferous lepidopteran pests in Taiwan. J. Invertebr. Pathol. 95: 71-76.

Kyei-Poku G., Gauthier D., van Frankenhuyzen K. 2008: Molecular data and phylogeny of Nosema infecting lepidopteran forest defoliators in the genera Choristoneura and Malacosoma. J. Euk. Microbiol. 55: 51-58.

Kyei-Poku G., Gauthier D., Schwarz R., van Frankenhuyzen K. 2011: Morphology, molecular characteristics and prevalence of a Cystosporogenes species (Microsporidia) isolated from Agrilus anxius (Coleoptera: Buprestidae). J. Invertebr. Pathol. 107: $1-10$.

Lee R.C., Williams B.A., Brown A.M., Adamson M.L., KeelING P.J., FAST N.M. 2008b: Alpha- and beta-tubulin phylog-

Received 8 January 2013 enies support a close relationship between the microsporidia Brachiola algerae and Antonospora locustae. J. Euk. Microbiol. 55: 388-392.

Lee S.C., Corradi N., Byrnes E.J., III., Torres-Martinez S., Dietrich F.S., Keeling P.J., Heitman J. 2008a: Microsporidia evolved from ancestral sexual fungi. Curr. Biol. 18: 1675-1679.

McKean P.G., Vaughan S., Gull K. 2001: The extended tubulin superfamily. J. Cell. Sci. 114: 2723-2733.

Nageswara Rao, S., Muthulakshmi, M., Kanginakudru, S. Nagaraju, J. 2004: Phylogenetic relationships of three new microsporidian isolates from the silkworm, Bombyx mori. J. Invertebr. Pathol. 86: 87-95.

Peyretaillade E., Biderre C., Peyret P., Duffieux F., Metenier G., Gouy M., Michot B., Vivares C.P. 1998: Microsporidian Encephalitozoon cuniculi, a unicellular eukaryote with an unusual chromosomal dispersion of ribosomal genes and a LSU rRNA reduced to the universal core. Nucl. Acids Res. 26: 3513-3520.

Peyretaillade E., El Alaoui H., Diogon M., Polonais V., Parisot N., Biron D.G., Peyret P., Delbac F. 2011: Extreme reduction and compaction of microsporidian genomes. Res. Microbiol. 162: 598-606.

Tamura K., Peterson D., Peterson N., Stecher G., Nei M., KumAR S. 2011: MEGA5: molecular evolutionary genetics analysis using maximum likelihood, evolutionary distance, and maximum parsimony methods. Mol. Biol. Evol. 28: 2731-2739.

Texier C., Vidau C., Vigues B., El Alaoui H., Delbac F. 2010: Microsporidia: a model for minimal parasite-host interactions. Curr. Opin. Microbiol. 13: 443-449.

Thompson J.D., Gibson T.J., Plewniak F., Jeanmougin F., HigGINS D.G. 1997: The CLUSTAL_X windows interface: flexible strategies for multiple sequence alignment aided by quality analysis tools. Nucl. Acids Res. 25: 4876-4882.

Tsai S.J., Huang W.F., Wang C.H. 2005: Complete sequence and gene organization of the Nosema spodopterae rRNA gene. J. Euk. Microbiol. 52: 52-54.

Tsai S.J., Lo C.F., Soichi Y., Wang C.H. 2003: The characterization of microsporidian isolates (Nosematidae: Nosema) from five important lepidopteran pests in Taiwan. J. Invertebr. Pathol. 83: 51-59.

Vossbrinck C.R., Debrunner-Vossbrinck B.A. 2005: Molecular phylogeny of the Microsporidia: ecological, ultrastructural and taxonomic considerations. Folia Parasitol. 52: 131-142.

Vossbrinck C.R., Maddox J.V., Friedman S., Debrunner-VossBRINCK B.A., Woese C.R. 1987: Ribosomal RNA sequence suggests microsporidia are extremely ancient eukaryotes. Nature 326: 411-414.

Wang L.L., Chen K.P., Zhang Z., Yao Q., Gao G.T., Zhao Y. 2006: Phylogenetic analysis of Nosema antheraeae (Microsporidia) isolated from Chinese oak silkworm, Antheraea pernyi. J. Euk. Microbiol. 53: 310-313.

Wittner M., Weiss L.M. 1999: The Microsporidia and Microsporidiosis. American Society for Microbiology Press, Washington, D.C., $553 \mathrm{pp}$.

Zhu F., Shen Z., Guo X., Xu X., Tao H., Tang X., Xu L. 2011: A new isolate of Nosema sp. (Microsporidia, Nosematidae) from Phyllobrotica armata Baly (Coleoptera, Chrysomelidae) from China. J. Invertebr. Pathol. 106: 339-342.

Zhu F., Shen Z., Xu X., Tao H., Dong S., Tang X., Xu L. 2010: Phylogenetic analysis of complete rRNA gene sequence of Nosema philosamiae isolated from the lepidopteran Philosamia cynthia ricini. J. Euk. Microbiol. 57: 294-296. 